Aim This multicentre observational study was conducted to confirm the observed retrospective findings prospectively in UK clinical practice.

Methods Retrospective data were collected in the 12 months prior to and prospective data for up to 12 months following omalizumab initiation. The primary endpoint was the change in mean daily OCS dosage (reported previously). Secondary endpoints included changes in mean exacerbation frequency (defined as requiring hospital admission or Accident and Emergency (A\&E) attendance and/or a course of OCS (dosage increase of at least $10 \mathrm{mg} /$ day for at least 3 days)), healthcare utilisation and missed days in education or at work.

Results 235 patients were enrolled in the study at end December 2013 in 22 UK centres. Data for interim analysis were examined from patients with 12 months of assessment at database lock (n $=85$, females, 54\%, mean $( \pm \mathrm{SD})$ age $44 \mathrm{yr}( \pm 13.2)$, mean $( \pm S D)$ duration of asthma $26 \mathrm{yr}( \pm 14.0))$. At the 16 weeks assessment $74 / 85$ (87\%) patients were classified as responders to omalizumab treatment. Comparing the 12 month periods prior to and following initiation of omalizumab, mean total exacerbations decreased by $51 \%$ from (mean, \pm SD) $4.25 \pm 2.73$ to 2.07 \pm 2.01 (mean difference 2.18, $\mathrm{p}<0.001$ ), while mean exacerbations involving hospital visits decreased by $61 \%$ from $1.52 \pm$ 2.00 to $0.59 \pm 1.25$ (difference $0.93, \mathrm{p}<0.001$ ). A\&E attendances were reduced from 54 to $19(\mathrm{p}<0.01)$ and inpatient hospitalisations from 85 to 36 ( $p<0.001)$. The percentage of average days absent from work or education due to sickness was more than halved in the 12 months pre and post omalizumab initiation reducing from $19.6 \%$ to $7.72 \%(n=27, p<0.05)$.

Conclusions The data prospectively confirms that omalizumab is associated with significant reduction in exacerbations, healthcare utilisation and societal burden in severe allergic asthma patients as was reported in the retrospective study.

\section{S94 A PROSPECTIVE STUDY INVESTIGATING ORAL CORTICOSTEROID (OCS) USE AND QUALITY OF LIFE IN OMALIZUMAB TREATED SEVERE ALLERGIC ASTHMA PATIENTS - RESULTS FROM AN INTERIM ANALYSIS OF THE APEX II STUDY}

${ }^{1} \mathrm{R}$ Chaudhuri, ${ }^{2} \mathrm{~A}$ Menzies-Gow, ${ }^{3} \mathrm{H}$ Khachi, ${ }^{4} \mathrm{~S}$ Hand, ${ }^{5} \mathrm{R}$ Gore, ${ }^{6} \mathrm{R}$ Niven. ${ }^{1}$ Gartnavel General Hospital, Glasgow, UK; ${ }^{2}$ Royal Brompton, London, UK; ${ }^{3}$ London Chest Hospital, London, UK; ${ }^{4}$ Prince Charles Hospital, Merthyr Tydfil, UK; ${ }^{5}$ Lister Hospital, Stevenage, UK; ${ }^{6}$ University Hospital of South Manchester, Manchester, UK

\subsection{6/thoraxjnl-2014-206260.100}

Background A previous retrospective study of UK clinical practice demonstrated that omalizumab reduced OCS burden.

Aim This multi-centre observational study was conducted to confirm the observed retrospective findings prospectively.

Methods Retrospective data were collected in the 12 months prior to omalizumab, while prospective data were collected at 16 weeks, 8 and 12 months following initiation. The primary endpoint was the change in mean daily OCS dose in the 12 months pre and post omalizumab initiation. Secondary endpoints included changes in ACT and AQLQ scores in the 12 months pre and post omalizumab initiation.

Results 235 patients were enrolled by the end December 2013 in 22 UK centres. Data for interim analysis were examined from patients who had 12 months assessment at database lock $(\mathrm{n}=85,54 \%$ females, mean $( \pm \mathrm{SD})$ age $44 \mathrm{yr}( \pm 13.2)$, mean $( \pm S D)$ duration of asthma $26 \mathrm{yr}( \pm 14.0))$. At the 16 weeks assessment $74 / 85(87 \%)$ patients were classified as responders to

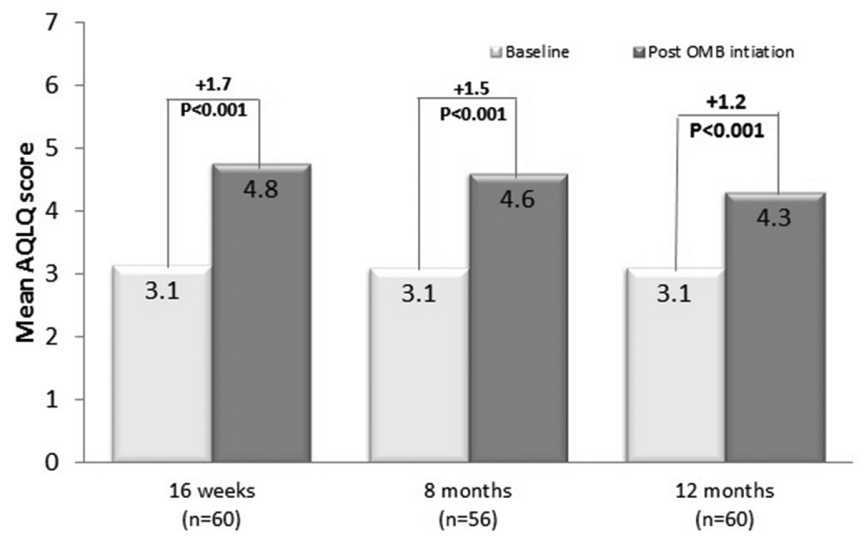

Abstract S94 Graph 1 Mean AQLQ score pre and post omalizumab initiation - ITT group

omalizumab treatment. At 12 months, mean daily OCS dose decreased by $25 \%(\mathrm{n}=85, \mathrm{p}<0.001)$ from $10.77 \mathrm{mg} /$ day $( \pm 7.87)$ to $8.08 \mathrm{mg} / \mathrm{day}( \pm 8.39)$ and $55 \%(\mathrm{n}=46 / 84)$ of patients stopped OCS. 71\% $(\mathrm{n}=60 / 85)$ of patients stopped or reduced OCS by $\geq 20 \%$. Comparing the 12 months periods prior to and following initiation of omalizumab, the mean ACT score improved from $9.8( \pm 4.8)$ to $14.2( \pm 5.2)(\mathrm{n}=75$, $\mathrm{p}<0.001)$ and the mean AQLQ score improved from 3.1 $( \pm 1.3)$ to $4.3(1.46)$ ( $\mathrm{n}=60, \mathrm{p}<0.001)$ [Graph 1].

Conclusions The data prospectively confirms that omalizumab is associated with statistically and clinically significant reduction in OCS and statistically and clinically significant improvement in asthma symptom control and quality of life.

\section{S95 DOUBLE-BLIND MULTI-CENTRE RANDOMISED CONTROLLED TRIAL OF VITAMIN D3 SUPPLEMENTATION IN ADULTS WITH INHALED CORTICOSTEROID-TREATED ASTHMA (VIDIAS)}

${ }^{1}$ AR Martineau, ${ }^{1}$ BD MacLaughlin, ${ }^{1}$ RL Hooper, ${ }^{1} \mathrm{NC}$ Barnes, ${ }^{1} \mathrm{DA}$ Jolliffe, ${ }^{2} \mathrm{AB}$ Choudhury, ${ }^{3}$ RK Rajakulasingam, ${ }^{3} \mathrm{~A}$ Bhowmik, ${ }^{4} \mathrm{DE}$ Simcock, ${ }^{1} \mathrm{~J}$ Grigg, ${ }^{5} \mathrm{CJ}$ Corrigan, ${ }^{5} \mathrm{CM}$ Hawrylowicz, ${ }^{1} \mathrm{CJ}$ Griffiths. 'Queen Mary University of London, London, UK; ${ }^{2}$ Queen's Hospital, Romford, UK; ${ }^{3}$ Homerton Hospital, London, UK; ${ }^{4}$ Royal London Hospital, London, UK; ${ }^{5}$ King's College London, London, UK

\subsection{6/thoraxjnl-2014-206260.101}

Introduction and objectives Asthma exacerbations are commonly precipitated by viral upper respiratory infections (URI). Vitamin $D$ insufficiency associates with susceptibility to URI in patients with asthma. A recent vitamin $\mathrm{D}$ trial in adults with asthma reported a trend towards reduced exacerbation risk in the intervention arm as a secondary outcome. Trials of vitamin D in adults with asthma with incidence of exacerbation and URI as primary outcome are lacking. We therefore conducted a multicentre randomised controlled trial of vitamin $\mathrm{D}_{3}$ supplementation in adults with asthma with co-primary outcomes of severe exacerbation and URI.

Methods Two hundred and fifty adults with inhaled corticosteroid (ICS)-treated asthma were allocated to receive six 2-monthly oral doses of $3 \mathrm{mg}$ vitamin $\mathrm{D}_{3}$ or placebo over one year. Co-primary outcomes were time to first severe exacerbation and time to first URI. Sub-group analyses were performed to determine whether effects of supplementation were modified by baseline vitamin $\mathrm{D}$ status or genotype for thirty-four single nucleotide polymorphisms in eleven vitamin D pathway genes. 
Results Participants were allocated to vitamin $\mathrm{D}_{3}$ vs. placebo in equal numbers; $82 \%$ were vitamin $\mathrm{D}$ insufficient at baseline. Vitamin $\mathrm{D}_{3}$ supplementation did not influence time to first severe exacerbation (aHR 1.02, 95\% CI 0.69-1.53, P = 0.91) or time to first URI (aHR $0.87,95 \%$ CI $0.64-1.16, \mathrm{P}=0.34$ ). The influence of vitamin $\mathrm{D}_{3}$ on co-primary outcomes was not modified by baseline vitamin $\mathrm{D}$ status or genotype. Of 16 prespecified secondary outcomes, only one showed a difference between arms: vitamin $\mathrm{D}$ supplementation induced a modest improvement in respiratory quality of life as evidenced by a reduction in mean total score for the St George's Respiratory Questionnaire at 2 months ( -3.9 points, $\mathrm{p}=0.005), 6$ months $(-3.7$ points, $\mathrm{p}=0.038)$ and 12 months $(-3.3$ points, $\mathrm{p}=0.060)$.

Conclusions Vitamin $\mathrm{D}_{3}$ supplementation did not influence time to exacerbation or URI in a population of adults with ICStreated asthma with a high prevalence of baseline vitamin D insufficiency.

\section{S96 BRONCHIAL THERMOPLASTY REDUCES PERIPHERAL BLOOD EOSINOPHILS IN SEVERE ASTHMA DEMONSTRATING SYSTEMIC EFFECTS OF A LOCALISED THERAPY}

DM Ryan, U Holmes, G McCumesky, RD Daly, K Hince, G Tavernier, S Fowler, RM Niven. University Hospital South Manchester, Manchester, UK

\subsection{6/thoraxjnl-2014-206260.102}

Introduction Severe Asthma, characterised by persistent symptoms despite maximal medical therapy, represents $5 \%$ of asthma cases. Bronchial Thermoplasty (BT) is a novel therapy, NICE approved for Severe Asthma patients uncontrolled despite step 4/5 of British Guideline on Asthma Management. BT delivers radiofrequency thermal energy to airways distal to the mainstem bronchi, permanently reducing airway smooth muscle mass. It is unknown whether treatment of smooth muscle hypertrophy impacts persistently upon systemic signs of allergic inflammation. Peripheral blood eosinophils (PBEs) are a marker of allergic inflammation in asthma. We asked: does BT modify signs of allergic inflammation as measured by PBEs and if so, does this effect persist over time?

Method A retrospective review of 15 consecutive Severe Asthma cases treated with BT was performed. Serial PBEs measured

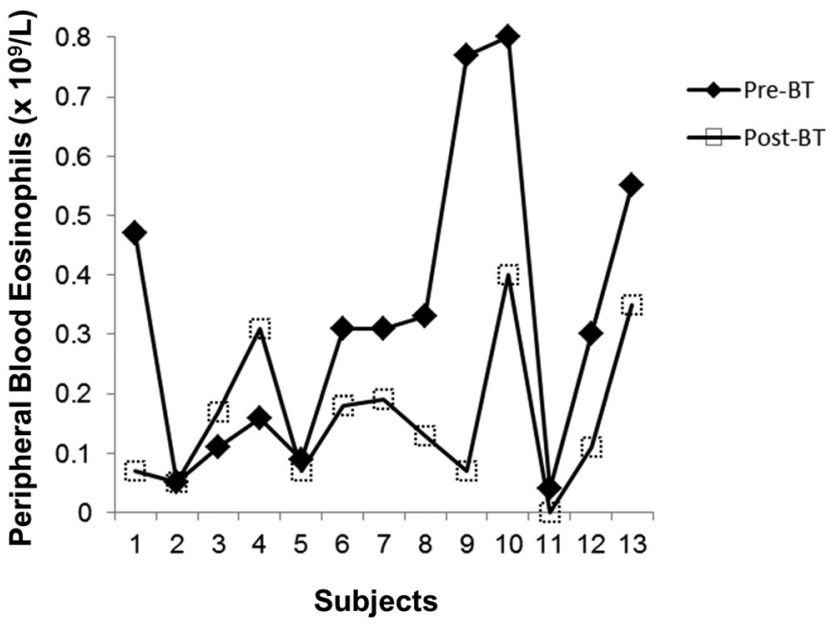

Abstract S96 Figure 1 before and up to 1 year after BT were compared. Blood eosinophil levels taken peri-procedure were excluded from analysis due to standard protocol concomitant steroid therapy. For time to first detectable high PBE all available post-BT PBE levels were assessed.

Results 13 patients had PBE data before and after BT, with an average of 9 and 12 serial PBE levels pre and post-BT respectively. Mean PBE 1 year pre-BT was $0.33 \times 10^{9} / \mathrm{L}$ falling to a mean of $0.16 \times 10^{9} / \mathrm{L} 1$ year post-BT $(\mathrm{p}<0.05)$ (see Figure). 9 of 13 patients had a fall in mean PBE, in 2 of 13 levels rouse and 1 of 13 mean PBEs were unchanged post-BT. In 6 patients who converted from normal to high PBE post-BT, average time to first high $\mathrm{PBE}\left(>0.4 \times 10^{9} / \mathrm{L}\right)$ was 7 months (range 1-13 months). In 5 patients (38\%) PBE remained within normal range persistently post BT.

Conclusion Severe Asthma patients undergoing BT had a significant reduction in average peripheral blood eosinophil levels from baseline. In over $1 / 3$ of cases this effect was persistent 1 year post procedure. These findings support the concept that BT not only reduces asthma-associated smooth muscle hypertrophy but impacts upon systemic markers of allergic inflammation.

\section{Mechanistic insights in acute lung injury}

\section{S97 LONG TERM SURVIVAL IN PATIENTS WHO UNDERGO OESOPHAGECTOMY IS LOWER IN PATIENTS WHO DEVELOP POST-OPERATIVE ACUTE RESPIRATORY DISTRESS SYNDROME}

${ }^{1}$ RCA Dancer, ${ }^{1} \mathrm{D}$ Parekh, ${ }^{2} \mathrm{GD}$ Perkins, ${ }^{1} \mathrm{DR}$ Thickett. ${ }^{1}$ University of Birmingham, Birmingham, UK; ${ }^{2}$ University of Warwick, Coventry, UK

\subsection{6/thoraxjnl-2014-206260.103}

Oesophagectomy is a complicated procedure with high risk of complications in the immediate post-operative period. We have previously shown that patients undergoing oesophagectomy have $\sim 25 \%$ risk of Acute Respiratory Distress Syndrome (ARDS) post op. Post-operative complications have been shown to decrease long term post-operative survival following major surgery. ${ }^{1}$ We hypothesised that long term survival would be reduced in patients who develop ARDS post oesophagectomy.

We analysed data from 55 patients recruited to the translational sub-study of the BALTI prevention trial. 26 of the 55 patients $(47 \%)$ died within 2 years of their operation.

Patients who died within two years of their oesophagectomy were more likely to have required ventilation for ARDS during their hospital admission. In addition, patients who survived less than two years were more likely to have developed a surgical complication (e.g. anastomotic leak, wound infection, chyle leak) post-op. There was no difference in age, lung function, BMI or cancer staging. Patients who did not survive more than 2 years post-op were more likely to be smoking at the time of the operation, but there was no difference in pack year smoking history between the two groups.

Perioperative markers of alveolar epithelial damage (PICCO EVLWI and PVPI), and the severity of both local (BAL CRP) and systemic inflammation (IL-17, ICAM-1, and TNFR-1/2) were associated with outcome.

In conclusion, complications during recovery from oesophagectomy have an adverse effect on the chances of long term survival. Development of strategies to reduce post-operative morbidity may improve long term outcomes. 